\title{
Mean Platelet Volume Could be a Possible Biomarker for Papillary Thyroid Carcinomas
}

\author{
Suleyman Baldane ${ }^{1 *}$, Suleyman H Ipekci ${ }^{1}$, Mehmet Sozen², Levent Kebapcilar ${ }^{1}$
}

\begin{abstract}
Thyroid cancer is the most prevalent endocrine cancer and is evident in nearly $5 \%$ of thyroid nodules. The correlation between mean platelet volume (MPV) and many other cancer types has been investigated previously. However, the correlation between papillary thyroid carcinoma (PTC) and MPV has not yet been studied in detail. The aim of this study was to examine whether MPV would be a useful inflammatory marker to differentiate PTC patients from cases of benign goiter and healthy controls. Preoperative MPV levels in patients with PTC were found to be significantly higher when compared with benign goiter patients and healthy controls ((respectively, 8.05 femtoliter ( $\mathrm{fl}$ ), $7.57 \mathrm{fl}, 7.36 \mathrm{fl}, \mathrm{p}=\mathbf{0 . 0 0 1})$. After surgical treatment of PTC patients, a significant decrease in MPV levels was seen $(8.05 \mathrm{fl}$ versus $7.60 \mathrm{fl}, \mathrm{p}=\mathbf{0 . 0 0 5})$. ROC analysis suggested 7.81 as the cut-off value for MPV (AUC=0.729, sensitivity 60\%, specificity 80\%). In conclusion, maybe changes in MPV levels can be used as an easily available biomarker for monitoring the risk of PTC in patients with thyroid nodules, enabling early diagnosis of PTC.
\end{abstract}

Asian Pac J Cancer Prev, 16 (7), 2671-2674

\section{Introduction}

Thyroid cancer is the most prevalent endocrine cancer and is seen in nearly 5\% of thyroid nodules (Pacini et al., 2006). Thyroid cancer incidence has rapidly increased during the past few decades, and the increase is nearly exclusively due to increases in the incidence of papillary thyroid carcinomas (PTCs), with no significant change for the other histotypes (Cramer et al., 2011; Pellegriti et al., 2013).

With their secretions of proinflammatory factors, chemokines, and growth factors, platelets play an important role in cancer diseases with an inflammatory background (George, 2000). Mean platelet volume (MPV) is a marker associated with functional changes in platelets, which can be easily detected with routinely used hemocytometers (Gasparyan et al., 2006; Wang et al., 2011). MPV indicates mean platelet size and reflects platelet production rate and stimulation (Threatte, 1993). Larger platelets are more metabolically and enzymatically active than smaller platelets (Mangalpally et al., 2010).

The correlation between MPV and many other cancer types has been investigated previously (Cho et al., 2013; Oge et al., 2013; Kemal et al., 2014; Kilincalp et al., 2014a; 2014b); however, the correlation between PTC and MPV has not yet been studied. The aim of this study is to examine whether MPV would be a useful inflammatory marker to differentiate PTC patients from benign goiters and healthy controls.

\section{Materials and Methods}

The present study was a retrospective study. The Ethical Committee and Institutional Review Board of Selcuk University Faculty of Medicine, where the study was conducted, approved the study design.

For this retrospective study, we reviewed the medical records of 152 patients who underwent a total thyroidectomy in our hospital between January 2013 and August 2014. Patients with diabetes mellitus, hypertension, chronic inflammatory disease or autoimmune disease, acute or chronic infection, other known malignancy, heart failure, myeloproliferative disorders, hepatic or renal disorders, taking anticoagulation medicine, and thyroid stimulating hormone (TSH) outside normal range were excluded from the study.

Thirty patients with exclusion criteria and 24 patients with missing data were not included in the study. Sixty-six out of 98 patients who underwent a total thyroidectomy had been diagnosed as having PTC $(n=66)$; the rest had benign goiters $(n=32)$. Twenty-eight hospital professionals with normal TSH and thyroid autoantibodies levels and their normal thyroid ultrasonographic examination results were included in the study as a healthy control group. As a result, all patients were divided into three groups as follows: Group $1(\mathrm{n}=66)$ were PTC patients, Group 2 $(n=32)$ were benign goiter patients, and Group $3(n=28)$ were healthy controls.

Each patient's medical record was independently 
Table 1. Demographic Features and Laboratory Results of the Study Groups

\begin{tabular}{lllll}
\hline & PTC $(\mathrm{n}=66)$ & Benign goiter $(\mathrm{n}=32)$ & Healthy controls $(\mathrm{n}=28)$ & $\mathrm{p}$ \\
\hline Age (years) & $42.0(24.0-64.0)$ & $45.0(19.0-76.0)$ & $37.6(27.0-50.0)$ & - \\
Gender (male/female) & $10 / 56$ & $5 / 27$ & $6 / 22$ & - \\
Smoking (yes/no) & $6 / 60$ & $4 / 28$ & $5 / 23$ & - \\
WBC $\left(\mathrm{x} 10^{9} / \mathrm{l}\right)$ & $7.98 \pm 2.11$ & $7.25 \pm 1.34$ & $7.04 \pm 1.57$ & 0.174 \\
Hb $(\mathrm{g} / \mathrm{dl})$ & $13.2(10.2-16.7)$ & $13.0(8.8-17.1)$ & $13.8(11.2-16.7)$ & $0.035^{*}$ \\
Platelets $\left(\mathrm{x} 10^{9} / \mathrm{l}\right)$ & $277 \pm 59.5$ & $281 \pm 58.4$ & $257 \pm 49.4$ & 0.145 \\
MPV (femtoliters,fl) & $8.05(7.06-10.60)$ & $7.57(5.86-8.81)$ & $7.36(5.59-8.97)$ & $0.001^{*}$ \\
\hline
\end{tabular}

*Comparisons needed to be $\mathrm{p}<0.017$ to be considered statistically significant; PTC, papillary thyroid carcinoma, WBC, white blood cell: Hb, hemoglobin; MPV, mean platelet volume

reviewed by two physicians of our department to ensure that exclusion criteria were accurately met in every candidate for enrollment in our study. Data collection included patient demographics, clinico-pathological parameters, and preoperative and postoperative hematological parameters.

For blood count analyses, samples were obtained after overnight fasting and between 7:30 AM and 9:00 AM. Preoperative data were obtained from the preoperative results of routine blood tests. Postoperative data were obtained from the routine control tests performed at postoperative 4-10 weeks while the patients were receiving L-thyroxine and their TSH levels were within normal limits. All blood samples were tested (as a part of their complete blood counts, CBC) using an automated hematology analyzer (Cell-Dyn 3200) (Abbott Diagnostics, Abbot Park, IL, USA) for measurement of MPV.In our clinic, the MPV reference range is determined as 7-11 fl.

\section{Statistical analyses}

Data were analyzed using the Statistical Package for

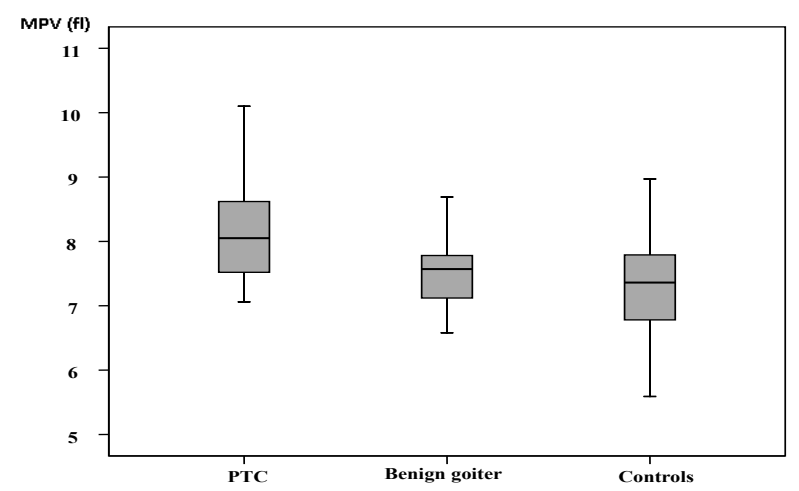

Figure 1. Median MPV in Groups. PTC: Papillary thyroid carcinoma, MPV: Mean platelet volume

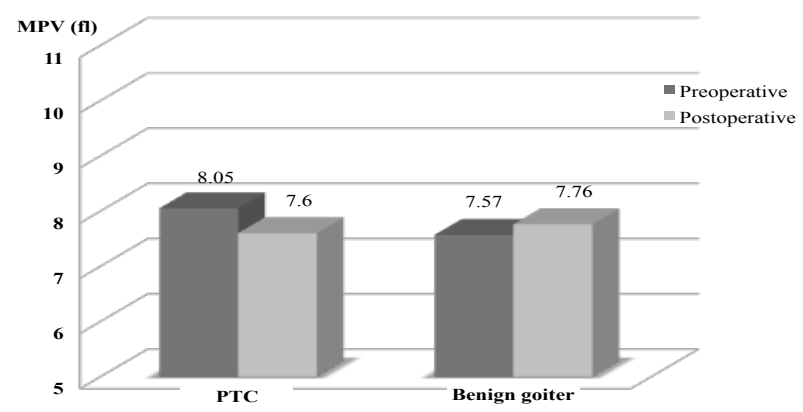

Figure 2. MPV Before and After Total Thyroidectomy in PTC and Benign Goiter Patient. PTC, papillary thyroid carcinoma, MPV, mean platelet volume
Social Sciences 16.0 for Windows (SPSS Inc., Chicago, IL). Continuous data were presented as mean \pm standard deviation or median (minimum-maximum), as appropriate. Proportions were compared using the Chi-square test. The Kruskal-Wallis test and the Mann-Whitney U test (Bonferroni correction) were used to compare the parameters of thyroid papillary cancer patients, benign goiter patients, and control subjects. The Wilcoxon test was used to compare the parameters of preoperative and postoperative MPV values in thyroid papillary cancer and benign goiter patients. Receiver-operating characteristic (ROC) curve analyses were performed to identify optimal cut-off values of the MPV level. The original significance level of 0.05 was adjusted so that individual comparisons needed to be $\mathrm{p}<0.017$ after Bonferroni correction.

\section{Results}

Demographic characteristics and preoperative laboratory parameters of the groups are given in Table 1 . There was no statistically significant difference among the groups regarding age; gender; smoking status; and white blood cell, hemoglobin, and platelet count (Table 1).

Preoperative MPV levels in patients with PTC were found to be significantly higher when compared with benign goiter patients and healthy controls (Table 1) (Figure 1). A significant difference was not observed between preoperative MPV levels of benign goiter patients and healthy controls (Table 1) (Figure 1).

After surgical treatment of PTC patients, a significant decrease in MPV levels was seen [8.05 fl (7.06-10.60 fl) versus $7.60 \mathrm{fl}(6.04-9.91 \mathrm{fl}), \mathrm{p}=0.005$ ] (Figure 2). However, in patients with benign goiters, a significant change was not observed in postoperative MPV levels [7.57 fl (5.818.81 ) versus $7.76 \mathrm{fl}$ (5.61-9.61), $\mathrm{p}=0.075$ ] (Figure 2$)$. The postoperative MPV levels of PTC patients and healthy controls were found to be comparable [7.60 fl (6.04-9.91) versus $7.36 \mathrm{fl}$ (5.59-8.97), $\mathrm{p}=0.402]$.

In the ROC curve analysis, the preoperative MPV presented $60 \%$ of sensitivity and $80 \%$ of specificity at the criterion $>7.81 \mathrm{fl}$ (area under the curve $($ AUC $)=0.729)$.

\section{Discussion}

This study demonstrated that PTC patients had significantly higher MPV values when compared with benign goiter patients and healthy controls. A significant drop in MPV levels has been demonstrated in PTC patients treated surgically. 
Many types of cancer emerge in the background of infection and inflammation. In the development and progression of cancer, inflammation is an essential and vital process (Kim et al., 2009). The biological effects of inflammation include increased cellular proliferation, angiogenesis, inhibition of apoptosis, and deficiencies in adaptation ability against oxidative stress (Shacter and Weitzman, 2002). Platelets have an important metabolic role in the pathogenesis of cancer by means of their angiogenic, metastatic, and proteolytic activities in the background of inflammation (Kisucka et al., 2006). MPV reflects platelet activity, and strong evidence indicates that MPV is an important biological variable and that larger platelets are more metabolically and enzymatically active than smaller platelets (Mangalpally et al., 2010). Previously, the association between many different types of cancer and MPV was investigated (Kilincalp et al., 2014; Kemal Y et al., 2014; Oge et al., 2013; Kilincalp et al., 2014; Cho et al., 2013); however, the correlation between PTC and MPV has not yet been analyzed. Recently published studies by Kemal et al. on patients with ovarian cancer (Kemal Y et al., 2014), Oge et al. on endometrial cancer patients (Oge et al., 2013), and two separate studies by Kilincalp et al. on patients with colorectal cancer (Kilincalp et al., 2014) and gastric cancer (Kilincalp et al., 2014) have demonstrated higher preoperative MPV values when compared with healthy controls; they have also indicated a significant decrease in MPV levels with surgical excision of tumors. In the present study, the correlation between PTC and MPV has been evaluated for the first time. Similar to previous studies, the present study has determined that PTC patients had significantly higher MPV values when compared with benign goiter patients and healthy controls; further, a significant drop in MPV values was detected after surgical treatment.

Malignant cells produce cytokines as IL-1, IL-6, and other growth factors, which stimulate the production of platelets. There is increasing evidence that tumors and endothelial cells are acted upon by vascular endothelial growth factor (VEGF), growth factors, and interleukins secreted by platelets (Sierko and Wojtukiewicz, 2004). This may contribute to the transport of VEGF and platelet derived growth factor (PDGF), which have been shown to function as a potent mitogen for different cell types (Sierko and Wojtukiewicz, 2004; Apte et al., 2004). In recent years, use of these anti-angiogenic drugs that target these pathways in the treatment of cancer is becoming more prevalent. These drugs can be effective on thrombocytic activity, which can be evaluated with MPV levels. If platelets have an important place in tumor angiogenesis, then MPV, which reflects thrombocytic activity, can be used as a marker for angiogenesis. Mutlu et al. investigated 74 patients with metastatic colon cancer and reported a significant decrease in MPV levels with the anti-angiogenic agent bevacizumab (Mutlu et al., 2012). Tuncel et al. analyzed 148 patients with colorectal cancer and reported that patients with metastatic disease had higher MPV values relative to the non-metastatic group; further, patients with higher MPV values among those treated with bevacizumab had shorter progression- free survival times and lower treatment response rates (Tuncel et al., 2014). The outcomes of this study can be interpreted as follows: increased platelet activity decreases the effectiveness of anti-angiogenic drugs. In recent years, anti-angiogenic drug use, mainly sorafenib therapy, in advanced PTC patients has been intensively investigated (Krajewska and Jarzab, 2014). In the years to come, new studies evaluating the correlation between anti-angiogenic treatment and MPV in PTC patients may be needed.

There are several limitations to our study. Our study is a retrospective study and it had a relatively small sample size.

In conclusion, our findings suggest that MPV may be used as a possible biomarker in the diagnosis of PTC patients. Maybe changes in MPV levels can be used as an easily available biomarker for monitoring the risk of PTC in patients with thyroid nodules, enabling early diagnosis of PTC. Further, a postoperative decrease in MPV levels might contribute to evaluating therapeutic effectiveness in PTC patients.

\section{References}

Apte SM, Fan D, Killion JJ, Fidler IJ (2004). Targeting the platelet-derivedgrowth factor receptor in antivascular therapy for human ovarian carcinoma. Clinical Cancer Research, 10, 897-90.

Cho SY, Yang JJ, You E, et al (2013). Mean platelet volume/ platelet count ratio in hepatocellular carcinoma. Platelets, 24, 375-77.

Cramer JD, Fu P, Harth KC, Margevicius S, Wilhelm SM (2010). Analysis of the rising incidence of thyroid cancer using the Surveillance, Epidemiology and End Results national cancer data registry. Surgery, 148, 1147-52.

Gasparyan AY, Ayvazyan L, Mikhailidis DP, Kitas GD (2011). Mean platelet volume: A link between thrombosis and inflammation? Curr Pharm Des, 17, 47-58.

George JN (2000). Platelets. Lancet, 355, 1531-39.

Kemal Y, Demiră̆ G, Ekiz K, Yücel I. Mean platelet volume could be a useful biomarker for monitoring epithelial ovarian cancer. J Obstet Gynaecol, 34, 515-18.

Kilincalp S, Coban S, Akinci H, et al (2014). Neutrophil/ lymphocyte ratio, platelet/lymphocyte ratio and mean platelet volume as potential biomarkers for early detection and monitoring of colorectal adenocarcinoma. Eur J Cancer Prev, [Epub ahead of print].

Kim DK, Oh SY, Kwon HC, et al (2009). Clinical significances of preoperative serum interleukin-6 and C-reactive protein level in operable gastric cancer. BMC Cancer, 9, 155.

Kisucka J, Butterfield CE, Duda DG, et al (2006). Platelets and platelet adhesion support angiogenesis while preventing excessive hemorrhage. Proc Natl Acad Sci USA, 103, 855-60.

Kilıncalp S, Ekiz F, Başar O, et al (2014). Mean platelet volume could be possible biomarker in early diagnosis and monitoring of gastric cancer. Platelets, 25, 592-94.

Krajewska J, Jarzab B (2014). Novel therapies for thyroid cancer. Expert Opin Pharmacother, 15, 2641-52.

Mangalpally KK, Siqueiros-Garcia A, Vaduganathan M, Dong JF, Kleiman NS, Guthikonda S (2010). Platelet activation patterns in platelet size subpopulations: differential responses to aspirin in vitro. J Thrombosis Thrombolysis, 30, 251-62.

Mutlu H, Berk V, Karaca H, et al (2012). Treatment regimen with bevacizumab decreases mean platelet volume in patients with metastatic colon cancer. Clin Appl Thromb Hemost, 18, 546-68. 
Oge T, Yalcin OT, Ozalp SS, Isikci T (2013). Platelet volume as a parameter for platelet activation in patients with endometrial cancer. J Obstet Gynaecol, 33, 301-14.

Pacini F, Schlumberger M, Dralle H, Elisei R, Smit JW, Wiersinga W; European Thyroid Cancer Taskforce (2006). European consensus for the management of patients with differentiated thyroid carcinoma of the follicular epithelium. Eur J Endocrinol, 154, 787-803.

Pellegriti G, Frasca F, Regalbuto C, Squatrito S, Vigneri $\mathrm{R}$ (2013). Worldwide increasing incidence of thyroid cancer: update on epidemiology and risk factors. $J$ Cancer Epidemiol, 2013, 965212.

Shacter E, Weitzman SA. Chronic inflammation and cancer (2002). Oncology, 16, 217-26.

Sierko E, Wojtukiewicz MZ (2004). Platelets and angiogenesis in malignancy. Seminars in Thrombosis and Hemostasis, 30, 95-108.

Threatte GA (1993). Usefulness of the mean platelet volume. Clinics in Laboratory Medicine, 13, 937-50.

Tuncel T, Ozgun A, Emirzeoglu L, Celik S, Bilgi O, Karagoz B (2014). Mean platelet volume as a prognostic marker in metastatic colorectal cancer patients treated with bevacizumab-combined chemotherapy. Asian Pac J Cancer Prev, 15, 6421-23.

Wang R-T, Li Y, Zhu X-Y, Zhang Y-N (2011). Increased mean platelet volume is associated with arterial stiffness. Platelets, 22, 447-51. 